\title{
Estabilidade Exponencial para um Problema de Transmissão para Equação da onda Amortecida
}

\author{
Félix Pedro Quispe Gómez \\ Departamento Acâdemico de Matemática, DAMAT - UTFPR, \\ 80230-901, Curitiba, PR \\ E-mail: felixgomez@utfpr.edu.br, felix12gomez@gmail.com
}

Resumo: Estudamos um problema de transmissão de um sistema de equações hiperbólicas formado por uma equação da onda amortecida tipo frição e uma equação da onda agindo cada uma numa parcela de seu domínio unidimensional. Para este modelo obtemos o decaimento exponencial utilizando a sistemática dos semigrupos e técnicas multiplicativas.

Palavras-chave: Semigrupos $C_{o}$, Estabilidade Exponencial, Problema Transmissão

\section{Introdução}

A preocupação principal será obter decaimento exponencial do seguinte sistema de equações diferenciais parciais. Considere um intervalo $] 0, L[\subset \mathbb{R}$ que se encontra dividido em dois subintervalos abertos, $] 0, l_{0}[\mathrm{e}] l_{0}, L[$. As equações diferenciais que governam o fenômeno são,

$$
\begin{aligned}
& \left.\partial_{t}^{2} u-\alpha \partial_{x}^{2} u+\gamma \partial_{t} u=0 \quad \text { em } \quad\right] 0, l_{0}[\times] 0,+\infty[ \\
& \left.\partial_{t}^{2} v-\beta \partial_{x}^{2} v=0 \quad \text { em } \quad\right] l_{0}, L[\times] 0,+\infty[
\end{aligned}
$$

com condições de transmissão,

$$
\begin{array}{rr}
u\left(l_{o}, t\right)=v\left(l_{o}, t\right), & t \in] 0,+\infty[ \\
\alpha \partial_{x} u\left(l_{o}, t\right)=\beta \partial_{x} v\left(l_{o}, t\right), & t \in] 0,+\infty[
\end{array}
$$

condições de fronteira,

$$
u(0, t)=v(L, t)=0, \quad t \in] 0,+\infty[
$$

condições iniciais,

$$
\begin{array}{lll}
u(x, 0)=u_{0}(x), & \partial_{t} u(x, 0)=u_{1}(x), & x \in] 0, l_{0}[ \\
v(x, 0)=v_{0}(x), & \partial_{t} v(x, 0)=v_{1}(x), & x \in] l_{0}, L[
\end{array}
$$

e finalmente as condições de compatibilidade,

$$
\begin{gathered}
u_{o}\left(l_{0}\right)=v_{o}\left(l_{0}\right), \quad u_{1}\left(l_{0}\right)=v_{1}\left(l_{0}\right) \\
\alpha \partial_{x} u_{o}\left(l_{o}\right)=\beta \partial_{x} v_{o}\left(l_{0}\right) .
\end{gathered}
$$

Vejamos a seguinte Figura 1 que ilustra o domínio do sistema em estudo, as constantes $\alpha$ e $\beta$ são distintas e a dissipação escolhida atua somente no domínio da função $u$. 


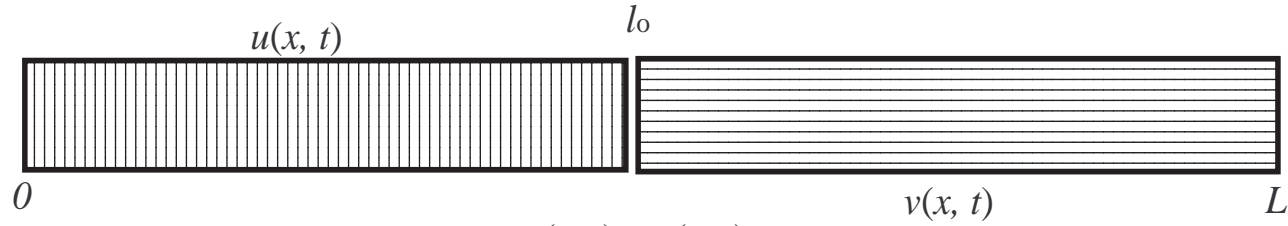

$u(x, t)=v(x, t)$

Condições de Transmissão

Figura 1: Domínio do problema de Transmissão

\section{Notações e Espaços Necessários}

Considere os seguintes espaços

$$
V_{1}=\left\{w \in H^{1}\left(0, l_{o}\right): w(0)=0\right\}, \quad V_{2}=\left\{v \in H^{1}\left(l_{o}, L\right): v(L)=0\right\} .
$$

Para os intervalos que formam o domínio adotamos a seguinte notação

$$
\left.I_{1}=\right] 0, l_{o}\left[\quad \mathrm{e} \quad I_{2}=\right] l_{o}, L[.
$$

Para a reformulação do problema consideramos as seguintes variáveis,

$$
U=\left(\begin{array}{c}
u \\
\partial_{t} u \\
v \\
\partial_{t} v
\end{array}\right), \quad U(0)=\left(\begin{array}{c}
u_{o} \\
u_{1} \\
v_{o} \\
v_{1}
\end{array}\right) \quad \text { e } \quad \mathcal{A}=\left(\begin{array}{cccc}
0 & I & 0 & 0 \\
\alpha \partial_{x}^{2} & -\gamma I & 0 & 0 \\
0 & 0 & 0 & I \\
0 & 0 & \beta \partial_{x}^{2} & 0
\end{array}\right)
$$

isto é,

$$
\partial_{t} U=\left(\begin{array}{c}
\partial_{t} u \\
\partial_{t}^{2} u \\
\partial_{t} v \\
\partial_{t}^{2} v
\end{array}\right)=\left(\begin{array}{c}
\partial_{t} u \\
\alpha \partial_{x}^{2} u-\gamma \partial_{t} u \\
\partial_{t} v \\
\beta \partial_{x}^{2} v
\end{array}\right), \quad \mathcal{A} U=\left(\begin{array}{cccc}
0 & I & 0 & 0 \\
\alpha \partial_{x}^{2} & -\gamma I & 0 & 0 \\
0 & 0 & 0 & I \\
0 & 0 & \beta \partial_{x}^{2} & 0
\end{array}\right)\left(\begin{array}{c}
u \\
\partial_{t} u \\
v \\
\partial_{t} v
\end{array}\right) .
$$

Logo o sistema correspondente ao problema de valor inicial (1.1)-(1.2) e (1.6) -(1.7), se transforma numa equação de evolução autônoma,

$$
\partial_{t} U=\mathcal{A} U, \quad t \geq 0, \quad U(0)=U_{o}
$$

A seguir construimos os espaços para esta reformulação. Considere o espaço

$$
\mathcal{H}=\left\{\left(\begin{array}{l}
u^{1} \\
v^{1} \\
u^{2} \\
v^{2}
\end{array}\right): u^{i} \in V_{i}, \quad v^{i} \in L^{2}\left(I_{i}\right), \quad i=1,2, \quad u^{1}\left(l_{0}\right)=u^{2}\left(l_{0}\right)\right\}
$$

com a seguinte norma

$$
\|U\|_{\mathcal{H}}^{2}=\int_{0}^{l_{0}} \alpha\left|\partial_{x} u^{1}\right|^{2}+\left|v^{1}\right|^{2} d x+\int_{l_{0}}^{L} \beta\left|\partial_{x} u^{2}\right|^{2}+\left|v^{2}\right|^{2} d x .
$$

O operador $\mathcal{A}$ de $\mathcal{H}$ em $\mathcal{H}$ possue o seguinte domínio,

$$
D(\mathcal{A})=\left\{\left(\begin{array}{l}
u^{1} \\
v^{1} \\
u^{2} \\
v^{2}
\end{array}\right) \in \mathcal{H}: u^{i} \in V_{i} \cap H^{2}\left(I_{i}\right), \quad v^{i} \in V_{i}, \quad i=1,2, \quad \alpha \partial_{x} u^{1}\left(l_{0}\right)=\beta \partial_{x} u^{2}\left(l_{0}\right)\right\} .
$$

Theorem 2.1 O problema de valor inicial (2.8) como o operador $\mathcal{A}$ linear esta bem colocado se e somente se $\mathcal{A}$ é o gerador infinitesimal de um semigrupo $S(t)$ de classe $C_{o}$. Neste caso a única solução de (2.8) esta dada por $U(t)=S(t) U_{o}$ para $U_{o}$ no domínio de $\mathcal{A}$.

Prova. A prova deste teorema se encontra em Goldstein [3]. 


\section{Existência de Soluções}

Utilizaremos o teorema de Hille-Yosida para semigrupos. Uma das hipóteses foi provada anteriormente. As únicas condições que estão faltando mostrar são as seguintes,

$$
\mathbb{R}^{+} \subset \rho(\mathcal{A}) \quad \text { e } \quad\left\|(\lambda I-\mathcal{A})^{-1}\right\|_{\mathcal{L}(\mathcal{H})} \leq \frac{1}{\lambda}, \quad \lambda \in \mathbb{R}^{+}
$$

onde a segunda é equivalente a,

$$
\left\|(\lambda I-\mathcal{A})^{-1} F\right\|_{\mathcal{L}(\mathcal{H})} \leq \frac{1}{\lambda}\|F\|_{\mathcal{H}}, \quad \lambda \in \mathbb{R}^{+} .
$$

Podemos representar $U=(\lambda I-\mathcal{A})^{-1} F$ e com esta notação temos a seguinte equivalência,

$$
(\lambda I-\mathcal{A})^{-1} F=U \quad \Leftrightarrow \quad \lambda U-\mathcal{A} U=(\lambda I-\mathcal{A}) U=F,
$$

e nos leva para seguinte equação em termos das coordenadas,

$$
\lambda\left(\begin{array}{c}
u^{1} \\
v^{1} \\
u^{2} \\
v^{2}
\end{array}\right)-\left(\begin{array}{c}
v^{1} \\
\alpha \partial_{x}^{2} u^{1}-\gamma v^{1} \\
v^{2} \\
\beta \partial_{x}^{2} u^{2}
\end{array}\right)=\left(\begin{array}{c}
f_{1} \\
g_{1} \\
f_{2} \\
g_{2}
\end{array}\right) .
$$

O sistema resolvente que nos permite mostrar a condições procuradas é,

$$
\begin{array}{rlrl}
\lambda u^{1}-v^{1} & =f_{1} & \text { em } & I_{1}, \\
\lambda v^{1}-\alpha \partial_{x}^{2} u^{1}+\gamma v^{1}=g_{1} & \text { em } & I_{1}, \\
\lambda u^{2}-v^{2}=f_{2} & \text { em } & I_{2}, \\
\lambda v^{2}-\beta \partial_{x}^{2} u^{2}=g_{2} & \text { em } & I_{2} .
\end{array}
$$

A etapa final será atuar em cada uma das equações que formam o sistema resolvente. Primeiro, multiplicamos pelos conjugados,

$$
-\alpha \overline{\partial_{x}^{2} u^{1}}, \quad \overline{v^{1}}, \quad-\beta \overline{\partial_{x}^{2} u^{2}}, \quad \overline{v^{2}}
$$

as equações (3.9), (3.10), (3.11) e (3.12) respectivamente. Logo somando esses resultados e utilizando as condições de transmissão (1.3) e (1.4) obteremos a seguinte desigualdade,

$$
\lambda\|U\|_{\mathcal{H}}^{2}+\gamma \int_{0}^{l_{o}}\left|v^{1}\right|^{2} d x \leq\|U\|\|F\|
$$

de onde obtemos,

$$
\lambda\left\|(\lambda I-\mathcal{A})^{-1} F\right\|=\lambda\|U\| \leq\|F\|
$$

$\log 0$

sendo o que desejavamos mostrar.

$$
\left\|(\lambda I-\mathcal{A})^{-1}\right\| \leq \frac{1}{\lambda}
$$

\section{Resultado de Decaimento Exponencial}

O nosso resultado de decaimento é uma aplicação do seguinte teorema,

Theorem 4.1 Seja $S(t)=e^{A t}$ um semigrupo de classe $C_{o}$ de contrações num espaço de Hilbert. Então $S(t)$ é exponencialmente estável se e somente se

$$
i \mathbb{R} \equiv\{i \beta, \beta \in \mathbb{R}\} \subseteq \rho(A)
$$

$e$

são válidas.

$$
\left\|(\lambda I-\mathcal{A})^{-1}\right\| \leq C, \quad \forall \quad \operatorname{Re}[\lambda] \geq 0
$$

Prova. A prova encontra-se nos trabalhos de Gearhart [2], Huang [4] e e Prüss [1]. 


\section{Resultado Principal}

Utilizando o mesmo procedimento para encontrar a estimativa (3.13) da seção anterior com $\lambda \in \mathbb{C}$ obtemos

$$
\operatorname{Re}[\lambda]\|U\|^{2}+\gamma \int_{0}^{l_{o}}\left|v^{1}\right|^{2} d x \leq\|U\|\|F\| .
$$

Em particular se tomamos $\lambda=i \beta$ temos que $\operatorname{Re}[\lambda]=0$ e obtemos a seguinte estimativa,

$$
\gamma \int_{0}^{l_{o}}\left|v^{1}\right|^{2} d x \leq\|U\|\|F\|
$$

onde a norma de $U$ em $\mathcal{H}$ é dada por,

$$
\|U\|_{\mathcal{H}}^{2}=\int_{0}^{l_{0}} \alpha\left|\partial_{x} u^{1}\right|^{2}+\left|v^{1}\right|^{2} d x+\int_{l_{0}}^{L} \beta\left|\partial_{x} u^{2}\right|^{2}+\left|v^{2}\right|^{2} d x .
$$

A idéia central, nesta implementação do Teorema 4.1 para com nosso problema em questão, é a recuperação da norma de $U$. Apartir de agora esse será nosso objetivo.

Multiplicando a equação (3.10) pelo conjugado $\overline{u^{1}}$ e integrando em $I_{1}$ obtemos,

$$
\lambda \int_{o}^{l_{o}} v^{1} \overline{u^{1}} d x+\alpha \int_{o}^{l_{o}}\left|\partial_{x} u^{1}\right|^{2} d x-\alpha u^{1}\left(l_{o}\right) \partial_{x} u^{1}\left(l_{o}\right)+\gamma \int_{o}^{l_{o}} v^{1} \overline{u^{1}} d x=\int_{o}^{l_{o}} g_{1} \overline{u^{1}} d x .
$$

$\mathrm{ou}$

$$
\alpha \int_{o}^{l_{o}}\left|\partial_{x} u^{1}\right|^{2} d x=-\lambda \int_{o}^{l_{o}} v^{1} \overline{u^{1}} d x-\gamma \int_{o}^{l_{o}} v^{1} \overline{u^{1}} d x+\int_{o}^{l_{o}} g_{1} \overline{u^{1}} d x+\alpha u^{1}\left(l_{o}\right) \partial_{x} u^{1}\left(l_{o}\right)
$$

logo utilizando a equação (3.9) temos,

$$
\begin{aligned}
\alpha \int_{o}^{l_{o}}\left|\partial_{x} u^{1}\right|^{2} d x= & -\frac{\lambda}{\bar{\lambda}} \int_{o}^{l_{o}}\left|v^{1}\right|^{2} d x-\gamma \int_{o}^{l_{o}} v^{1} \overline{u^{1}} d x \\
& +\int_{o}^{l_{o}} g_{1} \overline{u^{1}} d x-\frac{\lambda}{\bar{\lambda}} \int_{o}^{l_{o}} \bar{f}_{1} v^{1} d x+\alpha u^{1}\left(l_{o}\right) \partial_{x} u^{1}\left(l_{o}\right) .
\end{aligned}
$$

Utilizando a estimativa (3.13) e a desigualdade de Poincaré,

$$
\frac{\alpha}{2} \int_{o}^{l_{o}}\left|\partial_{x} u^{1}\right|^{2} d x \leq C\|F\|\|U\|+\left|\alpha u^{1}\left(l_{o}\right) \partial_{x} u^{1}\left(l_{o}\right)\right| .
$$

Multiplicando a relação (3.10) pela quantidade $x \overline{\partial_{x} u^{1}}$ e integrando temos,

$$
\begin{aligned}
\lambda \int_{0}^{l_{o}} x v^{1} \overline{\partial_{x} u^{1}} d x-\alpha \int_{0}^{l_{o}} x \partial_{x}^{2} u^{1} \overline{\partial_{x} u^{1}} d x & +\gamma \int_{0}^{l_{o}} x v^{1} \overline{\partial_{x} u^{1}} d x \\
& =\int_{0}^{l_{o}} x g_{1} \overline{\partial_{x} u^{1}} d x
\end{aligned}
$$

ou de forma equivalente,

$$
\begin{aligned}
\frac{\lambda}{\bar{\lambda}} \int_{0}^{l_{o}} x v^{1} \overline{\partial_{x} v^{1}} d x- & \frac{\alpha}{2} \int_{0}^{l_{o}} \partial_{x}\left|\partial_{x} u^{1}\right|^{2} d x+\gamma \int_{0}^{l_{o}} x v^{1} \overline{\partial_{x} u^{1}} d x \\
& =\int_{o}^{l_{o}} x g_{1} \overline{\partial_{x} u^{1}} d x-\frac{\lambda}{\bar{\lambda}} \int_{0}^{l_{o}} x v^{1} \overline{\partial_{x} f_{1}} d x .
\end{aligned}
$$

Especificamente teremos a seguinte situção,

$$
\begin{aligned}
-\frac{1}{2} \int_{o}^{l_{o}} x \partial_{x}\left|v^{1}\right|^{2} d x- & \frac{\alpha}{2} \int_{o}^{l_{o}} x \partial_{x}\left|\partial_{x} u^{1}\right|^{2} d x \\
& =-\gamma \int_{o}^{l_{o}} x v^{1} \overline{\partial_{x} u^{1}} d x+\int_{o}^{l_{o}} x g_{1} \overline{\partial_{x} u^{1}} d x-\frac{\lambda}{\bar{\lambda}} \int_{0}^{l_{o}} x v^{1} \overline{\partial_{x} f_{1}} d x .
\end{aligned}
$$


logo integrando por partes e maiorando teremos,

$$
I=\frac{l_{o}}{2}\left\{\left[v^{1}\left(l_{o}\right)\right]^{2}+\alpha\left[\partial_{x} u^{1}\left(l_{o}\right)\right]^{2}\right\} \leq \gamma \int_{o}^{l_{o}}\left|x v^{1} \overline{\partial_{x} u^{1}}\right| d x+C\|U\|\|F\| .
$$

Estimando o lado direito da desigualdade acima temos,

$$
\begin{aligned}
I & \leq C\|U\|\|F\|+\gamma l_{o}\left(\int_{o}^{l_{o}}\left|v^{1}\right|^{2}\right)^{1 / 2}\left(\int_{o}^{l_{o}}\left|\partial_{x} u^{1}\right|^{2}\right)^{1 / 2} \\
& \leq C\|U\|\|F\|+C_{1}\|U\|^{1 / 2}\|F\|^{1 / 2}\left\|\partial_{x} u^{1}\right\|_{L^{2}} .
\end{aligned}
$$

Da estimativa anterior (5.15) temos,

$$
I \leq C\|U\|\|F\|+C_{1}\|U\|^{1 / 2}\|F\|^{1 / 2}\left\{\left|u^{1}\left(l_{o}\right)\right|^{2}+\left|\partial_{x} u^{1}\left(l_{o}\right)\right|^{2}\right\}
$$

logo teremos a seguinte estimativa,

$$
\frac{1}{2} I \leq C_{2}\|F\|\|U\|+C_{3}\|U\|^{1 / 2}\|F\|^{1 / 2}\left|u^{1}\left(l_{o}\right)\right|^{2} .
$$

Utilizando a desigualdade de Gagliardo-Niremberg, teremos

$$
\left|u^{1}\left(l_{o}\right)\right| \leq\left\|u^{1}\right\|_{L^{\infty}} \leq C\left\|u^{1}\right\|_{L^{2}}^{1 / 2}\left\|\partial_{x} u^{1}\right\|_{L^{2}}^{1 / 2} \leq C_{\delta}\left\|u^{1}\right\|_{L^{2}}+\delta\left\|\partial_{x} u^{1}\right\|_{L^{2}} .
$$

Novamente da desigualdade anterior (5.16) obtemos a relação,

$$
\begin{aligned}
\left|\alpha u^{1}\left(l_{o}\right) \partial_{x} u^{1}\left(l_{o}\right)\right| & \leq \varepsilon_{o}\left|\partial_{x} u^{1}\left(l_{o}\right)\right|^{2}+C_{\varepsilon_{o}}\left|u^{1}\left(l_{o}\right)\right|^{2} \\
& \leq C \varepsilon_{o}\|U\|^{2}+\varepsilon_{o} C\|U\|\|F\|+C(\varepsilon, \delta)\left\|u^{1}\right\|_{L^{2}}+\varepsilon_{o}\left\|\partial_{x} u^{1}\right\|_{L^{2}}
\end{aligned}
$$

onde $\delta<\varepsilon_{o} / C_{\varepsilon_{o}}$.

Escrevendo de forma mais simplificada temos,

$$
\alpha u^{1}\left(l_{o}\right) \partial_{x} u^{1}\left(l_{o}\right) \leq C \varepsilon_{o}\|U\|^{2}+\varepsilon_{o} C\|U\|\|F\|+\widetilde{C_{\varepsilon}}\left\|u^{1}\right\|_{L^{2}}^{2}
$$

Considere $\lambda=i \beta$ e substitua na relação (3.9) para obter

$$
\begin{aligned}
\beta^{2} \int_{o}^{l_{o}}\left|u^{1}\right|^{2} d x & \leq 4 \int_{o}^{l_{o}}\left|v^{1}\right|^{2} d x+C\|F\|^{2} \\
& \leq 4\|U\|\|F\|+C\|F\|^{2}
\end{aligned}
$$

ou de maneira equivalente

$$
\int_{o}^{l_{o}}\left|u^{1}\right|^{2} d x \leq \frac{4}{\beta^{2}}\left[\|U\|\|F\|+C\|F\|^{2}\right]
$$

e quando $\beta \rightarrow \infty$, segue que existe $C_{o}$ tal que,

$$
\left\|u^{1}\right\|_{L^{2}}^{2}=\int_{o}^{l_{o}}\left|u^{1}\right|^{2} d x \leq C_{o}\|U\|\|F\|+d_{o}\|F\|^{2}
$$

portanto

$$
\frac{\alpha}{2} \int_{0}^{l_{o}}\left|\partial_{x} u^{1}\right|^{2} d x \leq \varepsilon_{o} C\|U\|^{2}+C_{\varepsilon}\|U\|\|F\|+C_{\varepsilon}\|F\|^{2}
$$

Utilizando a relação (5.14) obtemos

$$
\frac{1}{2}\left[\int_{0}^{l_{o}}\left|v^{1}\right|^{2}+\alpha\left|\partial_{x} u^{1}\right|^{2} d x\right] \leq C_{\varepsilon}\|U\|\|F\|+\varepsilon C\|U\|^{2}+C_{\varepsilon}\|F\|^{2} .
$$


Multiplicando a relação (3.12) por $(x-L) \partial_{x} u^{2}$, obtemos

$$
\begin{aligned}
\int_{l_{o}}^{L}\left[\left|v^{2}\right|^{2}+\beta\left|\partial_{x} u^{2}\right|^{2}\right] d x & \leq \frac{L-l_{o}}{2}\left\{\left[v^{2}\left(l_{o}\right)\right]^{2}+\beta\left[\partial_{x} u^{2}\left(l_{o}\right)\right]^{2}\right\}+\|F\|\|U\| \\
& \leq C\left\{\left|\partial_{x} u^{1}\left(l_{o}\right)\right|^{2}+\left|v^{1}\left(l_{o}\right)\right|^{2}\right\}+C\|U\|\|F\| \\
& \leq C \int_{o}^{l_{o}}\left|\partial_{x} u^{1}\right|^{2}+\left|v^{1}\right|^{2} d x+C\|U\|\|F\| \\
& \leq C \varepsilon_{o}\|U\|^{2}+C_{\varepsilon}\|U\|\|F\|+C_{\varepsilon}\|F\|^{2} .
\end{aligned}
$$

Das equações (5.19) e (5.20) e levando em consideração a norma de $U$ temos

$$
\frac{1}{2}\|U\|^{2} \leq C \varepsilon_{o}\|U\|^{2}+C_{\varepsilon}\|U\|\|F\|+C_{\varepsilon_{o}}\|F\|^{2}
$$

a seguir restringimos a constante $C \varepsilon_{o}<1 / 4$ e disto obteremos,

$$
\frac{1}{8}\|U\|^{2} \leq C_{\varepsilon}^{2}\|F\|^{2} \quad \text { e } \quad\|U\| \leq \widetilde{C_{\varepsilon}}\|F\|
$$

de onde resulta,

$$
\left\|(i \beta I-\mathcal{A})^{-1} F\right\| \leq C\|F\|, \quad \forall \quad \beta \in \mathbb{R},
$$

$\log \mathrm{O}$

$$
\left\|(i \beta I-\mathcal{A})^{-1}\right\| \leq C, \quad \forall \quad \beta \in \mathbb{R},
$$

existe o decaimento exponencial.

\section{Referências}

[1] Prüss, J.; On the Spectrum of $C_{0}$-semigroups, Trans. of the American Mathematical Society (2), vol. 284, pp. 847-857, (1984).

[2] Gearhart, L.; Spectral Theory for the Contractions Semigroups on Hilbert Spaces, Trans. of the American Mathematical Society (1), vol. 236, pp. 385-349, (1978).

[3] Goldstein, J. A.; Semigroups of Linear Operator and Applications, Oxford University Press, (1985).

[4] Huang, F.; Characteristic Conditions for Exponential Stability of the Linear Dynamical Systems in Hilbert Spaces, Annals of Differential Equations, vol 1 (1), pp. 43-56, (1985).

[5] Dautray, R. \& Lions, J.-L.; Mathematical Analysis and Numerical Methods for Science and Technology, Vol. 1,2 and 4, Springer-Verlag, Berlin, (1990).

[6] Athanasiadis, C. \& Stratis, I. G.; On Some Elliptic Transmission Problems, Annales Polonici Mathematici, LXIII.2, (1996).

[7] Neerven, J. V.; The Asymptotic Behaviour of Semigroups of Linear Operators, Operator Theory; vol 88. Berlin, Birkhäuser, (1996).

[8] Muñoz Rivera, J. E. \& Vila Bravo, J. C.; Asymptotic Stability of Semigroups Associated to the Linear Weak Dissipative Systems, Mathematical and Computer Modelling, vol. 40, (4), pp. 387-392, (2004).

[9] Liu, Z. \& Liu, Z.; Semigroups Associated with Dissipative Systems, CRC Research Notes Mathematics, 398 (3), Chapman \& Hall, (1999)

[10] Chen, S. \& Liu, K. \& Liu, Z.; Spectrum and Stability Elastic System with Global or Local Kelvin-Voigt Damping, SIAM Journal of Applied Mathematics Vol. 59(2), pp. 651$668,(1998)$. 